\title{
Inflammatory pseudotumours of the paranasal sinuses
}

\author{
Vivek Sasindran · A. Ravikumar · K. Senthil · L. Somu
}

\begin{abstract}
Inflammatory pseudotumors are clinocopathologically distinctive but biologically controversial entities. They have been described in the lungs, abdomen, retroperitoneum and extremities, but rarely affect the head and neck region. This report is of 2 cases of pseudo tumor of the paranasal sinuses with varied clinical presentation and successful outcome following treatment.
\end{abstract}

\section{Introduction}

Chronic inflammatory disorders of the nose and paranasal sinuses have been described very often in different clinical settings. Most often the offending pathogen is easily demonstrable and can be cultured. However certain chronic inflammatory conditions cause pseudotumors that present with symptoms due to their mass effect without any signs or symptoms attributable to the underlying inflammatory condition. These lesions have no demonstrable organisms to culture nor have any typical histopathological picture.

\footnotetext{
V. Sasindran ${ }^{1} \cdot$ A.Ravikumar $^{2}(\bowtie) \cdot$ K. Senthil ${ }^{3}$ • L. Somu ${ }^{4}$ ${ }^{1}$ Post Graduate

${ }^{2}$ Professor \& Head of Dept

${ }^{3}$ Associate Professor

${ }^{4}$ Assistant Professor

Dept of ENT, Head \& Neck Surgery

Sri Ramachandra Medical College \& Research Institute (DU)

Porur, Chennai - 600 116, India

E mail: entsrmc@yahoo.co.in
}

\section{Case report 1}

30 year old male presented with swelling over right side of nose of one year duration, gradual in onset and progressive in nature. It was associated with nasal block, nasal discharge, pain and headache. He had four episodes of spontaneous bleeding from right nostril, the last episode requiring 8 units of blood transfusion. He had no history of weight loss and loss of appetite. On examination, patient was pale and emaciated. Externally, a $3 \times 4 \mathrm{~cm}$ smooth swelling was seen bulging the right ala and lateral wall of the nose extending superiorly upto the level of the right nasal bone (Fig. 1). Anterior Rhinoscopy showed an irregular mass occupying the whole of the right nasal cavity. It was partly extruding out of the anterior nares pushing the septum to the left. Serous blood stained discharge was present within the nasal cavity. Probe could not be passed around the swelling and there was no bleeding on touch. Post nasal examination showed irregular mass extending to the right choana and abutting the right eustachian tube orifice. The left Eustachian tube orifice was free. Routine haematological investigations were normal. Mantoux test was negative and chest $\mathrm{X}$-ray was unremarkable. CT scan of the paranasal sinuses revealed a homogenous soft tissue mass involving the right maxillary sinus, nasal cavity, ethmoid, frontal and sphenoid sinus on the same side and blocking to the choana (Fig. 2). There was also evidence of breach of lamina papyracea and the nasal septum. A provisional diagnosis of inverted papilloma was made. Nasal mass was biopsied and sent for histopathological examination. In the immediate post operative period patient had profuse post nasal bleeding necessitating post nasal packing. The HPE report of the biopsied specimen was inconclusive. A complete excision of the nasal mass under general anaesthesia was planned, digital substraction angiogram performed revealed minimal vascularity with feeding vessels from the maxillary artery. Selective embolization with gel foam was carried out.

The patient was posted for a right partial maxillectomy $72 \mathrm{hrs}$ after the embolization with adequate blood reserved. 
Fig. 1 External swelling of the right ala and lateral wall of the nose extending superiorly upto the level of the right nasal bone

Fig. 2 CT scan of the paranasal sinuses

Fig. 3 Right medial maxillectomy

Fig. 4 Histopathological examination suggesting pseudotumor
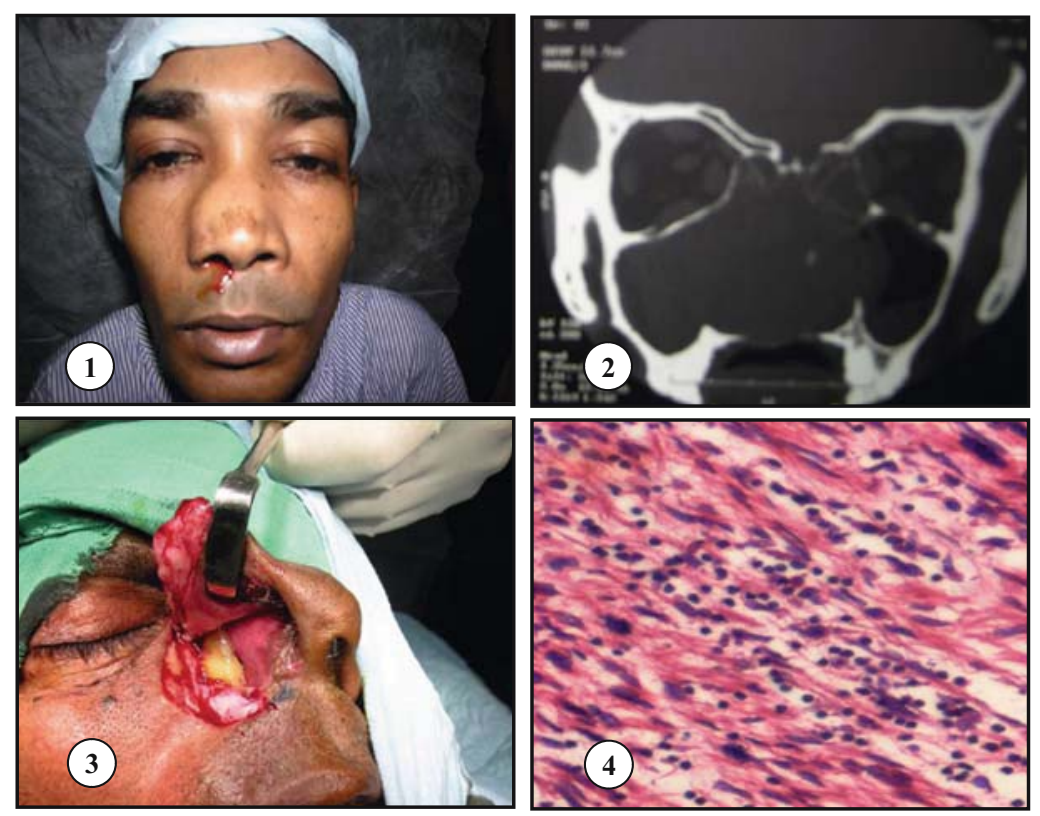

Lateral rhinotomy incision was made and periostium over the nasal bone and frontal process of the maxilla were elevated. Nasal bone on the right side was removed for adequate exposure and provided enough space to control bleeding. There was a large mass extending from the right nasal cavity on to the right maxillary antrum and involving the anterior and posterior ethmoidal sinuses. The ostia of the sphenoid as well as the frontal sinuses were blocked. The mass was extending posteriorly into the choana and hanging in the nasopharynx. There was no attachment with the septum and floor of the nose. Tissue bits were sent for frozen section which revealed only necrotic tissue. The whole mass was removed. Mass was confined to the mucosa with no evidence of bone destruction. Ethmoidal cells were cleared of disease. There was a breach of the lamina papyracea in the anterior part just behind the attachment of the middle turbinate with prolapse of orbital fat. Sphenoid sinus was opened and the polypoidal tissue was removed. The frontal sinus ostium was located and was found to be patent. Tissue from the posterior wall and the floor of the maxillary antrum were sent to assess for clearance of disease. Anterior and post nasal packing was done and the wound closed in layers. Four units of packed cells were transfused during the procedure. In the immediate post operative period patient had right periorbital edema which subsided on the third post operative day. HPE report was suggestive of inflammatory pseudo tumor probably of tuberculous etiology. However no acid fast bacilli were demonstrated histologically. Patient has been receiving Anti-tuberculous therapy and is on regular follow up. He is asymptomatic and nasal endoscopy done 6 months postop did not reveal any evidence of disease.

\section{Case report 2}

57 year old male presented with blocking of right nostril of six months duration. There was no associated bleeding from nose. Patient had similar complaints 2 years back and was diagnosed to have nasal mass and underwent Right medial maxillectomy for excision of nasal tumor. However the histopathological reports were not available. On examination, well healed lateral rhinotomy scar of the previous surgery was visible. Anterior Rhinoscopy revealed a pale looking mass $2 \times 2 \mathrm{~cm}$ occupying the right nasal cavity. The middle turbinate could not be visualized. The mass was sensitive to touch but did not bleed on touch. The probe could be passed all around. Post nasal examination revealed a smooth pale swelling abutting the right choana and the Eustachian tube orifice. CT scan of the paranasal sinuses revealed a homogenous opacity occupying the right nasal cavity with extension into the right maxillary antrum and partly into the nasopharynx. Haematological investigations were within normal limits and the patient had no other co-morbid conditions. Right medial maxillectomy was done under general anaesthesia (Fig. 3). Lateral rhinotomy incision made and the whole mass was removed in toto from the nasal cavity, nasopharynx and the maxillary antrum. The middle and the inferior turbinate were found to be removed as a part of the earlier surgery. Post operative period was uneventful. Histopathological examination revealed myofibroblastic spindle cells with lymphocytic infiltration, plasma cells and inflammatory cells suggestive of inflammatory pseudotumor (Fig. 4). Patient was followed up for 6 months with no evidence of recurrence. 


\section{Discussion}

Inflammatory pseudo tumour is a distinctive but biologically controversial entity that was originally described as a non-neoplastic lesion in the lung [1]. The condition was first described in 1905 by Birch-Hirschfield. The term pseudo tumour was coined because these lesions mimic expansive, invasive, malignant tumours both clinically and radiologically. It is also known as inflammatory myofibroblastic tumour. The aetiology and pathogenesis of these tumours still remain unknown. However, various stimuli may act as triggers for its development, such as unrecognized microorganisms, minor trauma, smoking and chronic irritation caused by cocaine abuse [2]. According to Williams et al, a localized derangement in the immune response after the initial insult may be an underlying mechanism [3]. These tumours have been reported to occur in every site in the body. In the head and neck region, it is commonly found in the orbit, nasal cavity, nasopharynx, the maxillary sinuses, the larynx, and the trachea [1]. In the nasal cavity and the paranasal sinuses, the patients present with complaints of unilateral nasal block, epistaxis, pain or local swelling. The clinical or endoscopic findings may demonstrate tumefaction covered by normal mucosa, a polyp, edematous mucosa, hypertrophic concha, or a bloody nasal discharge [4]. Inflammatory pseudo tumours located in the paranasal sinuses are usually associated with at least one sinus wall destruction. Inflammatory pseudo tumors of the head and neck region are generally not associated with non-specific systemic symptoms such as unexplained fever, weight loss and laboratory abnormalities. CT san and / or MRI of inflammatory pseudo tumours in the head and neck region often suggest infiltrative growth and can mimic meningioma, malignant neoplasms, Wegner's granulomatosis and sarcoidosis. Histologically these tumors are composed of myofibroblastic spindle cells, admixed with a prominent infiltrate of lymphocytes, plasma cells and acute inflammatory cells. Three basic histological patterns have been described, namely: (1) myxoid/vascular pattern, resembling inflammatory granulation tissue; (2) compact spindle cell pattern with fascicular and or storiform areas and variation of cellular density; and (3) hypocellular pattern, densly collagenised and reminiscent of a fibrous scar [1]. Immunochemistry confirms the myofibroblastic phenotype of the spindle cells.

Treatment and clinical outcome are generally favourable. The frequency of local recurrence is about $25 \%$. Radical excision is curative in more than $90 \%$ of the extrapulmonary inflammatory pseudo tumours, including head and neck. High dose oral corticosteroids [5], chemotherapy has been administered successfully. Radiation therapy has been advised in patients with recurrence and also patients who have medical contraindications to prolonged use of corticosteroids $[6,7]$. Metastasis to lung and brain has been reported. A case of pseudo tumour of the paranasal sinus with fatal outcome as result of extensive intracranial spread of the tumour has also been reported [8].

The differential diagnosis includes tumors such as sarcomatoid carcinoma, high grade angiosarcoma with spindle cell areas, leiomyosarcoma and fibrosarcoma. When myofibroblasts are set in a loose or myxoid stroma, the histological pattern may be indistinguishable from nodular faciitis [9].

In this article we have highlighted two cases of inflammatory pseudotumors with varied clinical presentations. Inflammatory tumours should be diagnosed early and radical excision should be done or else they can even spread intracranially when the outcome can be fatal. It is mandatory to follow up these patients as there is a high incidence of recurrence.

\section{References}

1. Coffin CM, Watterson J, Priest JR et al (1995) Extrapulmonary inflammatory myofibroblastic tumor (inflammatory pseudotumor). A clinicopathologic and Immunohistochemical study of 84 cases. American Journal of Surgical Pathology 19:859-872

2. Wenig BM, Devaney K, Bisceglia M (1995) Inflammatory moyfibroblastic tumor of the larynx: a clinicopathological study of eight cases simulating a malignant spindle cell neoplasm. Cancer 76:2217-2229

3. Williams SB, Foss RD, Ellis GL (1992) Inflammatory pseudotumors of the major salivary glands: clinicopathologic and Immunohistochemical anlysis of six cases. American Journal of Surgical Pathology 16:896-902

4. Ruaux C, Noret P, Godey B (2001) Inflammatory pseudotumor of the nasal cavity and sinuses. Journal of Laryngol Otol 115:563-566

5. Harr DL, Quencer RM, Abrams GW (1982) Computed tomography and ultrasound in the evaluation of orbital infection and pseudotumor. Radiology 142:395-401

6. Keen M, Cho HT, Savetsky L (1986) Pseudotumor of the larynx: an unusual cause of airway obstruction. Otolaryngology Head and Neck Surgery 94:243-246

7. Orcutt JC, Garner A, Henk JM, Wright JE (1983) Treatment of idiopathic inflammatory orbital pseudotumors by radiotherapy. British Journal of Ophthalmology 67:570-574

8. N Gale, N Zidar, J Podboj, M Volavsek, B Luzar (2003) Inflammatory myofibroblastic tumour of paranasal sinuses with fatal outcome: reactive lesion or tumour? Journal of Clinical Pathology 56:715-717

9. Shek AWH, Samman N (1996) Inflammatory Pseudotomour of the mouth and maxilla. Journal of Clinical Pathology 49: 164-167 